\title{
A Simulated Investigation of Ductile Response of GaAs in Single-Point Diamond Turning and Experimental Validation
}

\author{
Pengfei Fan ${ }^{1} \cdot$ Fei Ding ${ }^{1} \cdot$ Xichun Luo $^{1}$ (D) Yongda Yan ${ }^{2} \cdot$ Yanquan Geng $^{2} \cdot$ Yuzhang Wang $^{2}$
}

Received: 28 June 2020 / Revised: 23 September 2020 / Accepted: 9 October 2020 / Published online: 13 November 2020

(c) The Author(s) 2020

\begin{abstract}
In this paper, molecular dynamic (MD) simulation was adopted to study the ductile response of single-crystal GaAs during single-point diamond turning (SPDT). The variations of cutting temperature, coordination number, and cutting forces were revealed through MD simulations. SPDT experiment was also carried out to qualitatively validate MD simulation model from the aspects of normal cutting force. The simulation results show that the fundamental reason for ductile response of GaAs during SPDT is phase transition from a perfect zinc blende structure (GaAs-I) to a rock-salt structure (GaAs-II) under high pressure. Finally, a strong anisotropic machinability of GaAs was also found through MD simulations.
\end{abstract}

Keywords Molecular dynamic simulation $\cdot$ Single-point diamond turning $\cdot$ Gallium arsenide $\cdot$ Anisotropy $\cdot$ Ductile response

\section{Introduction}

The last few years have seen a wide exploitation of singlecrystal gallium arsenide (GaAs) in photoemitter devices [1], microwave devices [2], hall elements [3], solar cells [4], wireless communications [5], as well as quantum computation [6-8] due to its superior material properties such as higher temperature resistance, and higher electronic mobility and energy gap that outperforms silicon [9-12]. Ultraprecision multiplex $2 \mathrm{D}$ or $3 \mathrm{D}$ free-form nanostructures are often required on GaAs devices, such as radio frequency power amplifiers and switches used in 5G smart mobile wireless communications [13-15]. Currently, lapping [16, 17] and chemical-mechanical polishing [18-21] have been employed to successfully fabricate planar GaAs wafers. However, they are not competent for the fabrication of $2 \mathrm{D}$ or 3D nanostructures. Recently, focused ion beam (FIB) machining has been used to fabricate a hemispherical cavity with highly directional emission on a GaAs workpiece

Xichun Luo

xichun.luo@strath.ac.uk

1 Centre for Precision Manufacturing, DMEM, University of Strathclyde, Glasgow, UK

2 Center for Precision Engineering, Harbin Institute of Technology, Harbin, People's Republic of China
[22]. However, this approach is not viable for mass production for future commercialization due to the low material removal rate.

In this regard, single-point diamond turning (SPDT) [23-28] becomes a good candidate due to its capability of mass production of 2D and 3D nanostructures with high form accuracy in a single pass. Through establishing machining parameters to meet brittle-to-ductile transition condition, some researchers [29-31] have already successfully obtained nano-smooth machined surfaces on GaAs although it is regarded as a difficult-to-machine brittle material, attributing to its low elastic modulus and fracture toughness. However, so far, the underlying mechanisms of material ductile response and removal during SPDT of single-crystal GaAs have not yet been revealed. This has significantly hampered the development of the SPDT process for the manufacturing of 3D nanostructure on GaAs workpiece.

Molecular dynamic (MD) simulation has been proven to be a reliable theoretical approach to overcome the restriction of experimental observation to study ultra-precision machining brittle materials and has undergone prolific development in recent years [32]. It is capable of simulating structural characteristics of the work material at the atomic scale, which makes it possible to predict the motion and behavior of material atoms. Consequently, some fundamental issues related to nanomachining have been effectively investigated 
by using the MD approach. For example, Goel et al. [33] firstly revealed that the root cause of ductile response in machining cubic silicon carbide (3C-SiC) was an order-todisorder transition, i.e., the sp3-to-sp2 structural transformation, during SPDT by using the MD simulation. Subsequently, Xiao et al. [34] employed MD simulation to find that the combination of high-pressure phase transformation (HPPT) and dislocation plasticity was the origin of ductile response during SPDT of $6 \mathrm{H}-\mathrm{SiC}$. Additionally, Dai et al. [35] used MD simulation to uncover that the diamond cutting tool with a negative rake angle could experience a lower cutting resistance than the cutting tool with a positive rake angle during machining of single-crystal silicon. Likewise, Chavoshi et al. [36] presented plastic flow behavior of single-crystal silicon in stagnation region during SPDT at elevated temperature by displacement vectors analysis. Recently, Yi et al. [37] carried out a study of MD simulation of nanoscratching process of GaAs using a rigid spherical abrasive tool. The work did not investigate the reason of ductile response, temperature distribution of the cutting zone, and did not carry out experimental validation using a geometric cutting tool. In a word, these works show that MD simulation is a very effective method for investigating the removal mechanism of hard and brittle materials. Therefore, this paper employed MD approach to systematically elucidate an insight of material-removal mechanism for SPDT of single-crystal GaAs along with cutting forces of the diamond cutting tool, visualized temperature distribution of the cutting zone, structural changes of the machined GaAs workpiece, and the anisotropic machinability. In addition, SPDT experiment was conducted to verify the MD simulation results qualitatively. Further SPDT of single-crystal GaAs trial guided by the findings has verified the effectiveness of this simulated study.

\section{MD Simulation and SPDT Experimental Setups}

\subsection{Simulation Methodology}

\subsubsection{Simulation Model}

In this study, an orthogonal MD simulation model for SPDT of single-crystal GaAs, as illustrated in Fig. 1, was established and implemented by using an open source code large-scale atomic/molecular massively parallel simulator (LAMMPS) [38]. The visualization of simulation results was performed by using visual molecular dynamics (VMD) and open visualization tool (Ovito) software [39]. In MD cutting model, the diamond turning tool has a negative rake angle of $-25^{\circ}$, a clearance angle of $10^{\circ}$, and a tool nose radius of $2 \mathrm{~nm}$, as shown in Fig. 1b. Its size is $5.8 \mathrm{~nm} \times 5.0 \mathrm{~nm}$ $\times 2.8 \mathrm{~nm}$. To simplify MD simulation and save computational time, the diamond cutting tool was considered as a rigid body without wear and the machining took place with a velocity of $200 \mathrm{~m} / \mathrm{s}$ on the $\left(\begin{array}{lll}0 & 0 & 1\end{array}\right)$ surface along the $\left[\begin{array}{lll}1 & 1 & 0\end{array}\right]$ direction in this study. The dimension of the single-crystal GaAs substrate was $21 \mathrm{~nm} \times 10 \mathrm{~nm} \times 13.4 \mathrm{~nm}$ containing Newton atoms, thermostat atoms, and boundary atoms, as shown in Fig. 1a, whose initial temperature was maintaining at $300 \mathrm{~K}$ by using the Nose-Hoover method [40] running for $50 \mathrm{ps}$. Periodic boundary condition was applied in the $X$ and $Z$ directions. The simulation was carried out by a depth of cut of $2 \mathrm{~nm}$ and a cutting distance of $16 \mathrm{~nm}$ and was controlled under the NVE ensemble. The detailed simulation parameters are shown in Table 1.

\subsubsection{Adoption of Potential Energy Function}

In MD simulation, the force field was made of a hybrid potential function. The $\mathrm{C}-\mathrm{C}$ atomic interaction in the diamond machining tool was described by the classic Tersoff potential function, which is consistent with the majority of published work on MD simulation of brittle materials $[32,41]$. The interaction of single-crystal GaAs workpiece ( $\mathrm{Ga}-\mathrm{Ga}$, As-As, and $\mathrm{Ga}-\mathrm{As}$ ) was calculated using the updated bond order potentials (BOP) function based on quantum mechanical theory. BOP function, as shown in Eq. (1), is especially suitable for governing the gallium arsenide system because it considers an electron counting potential, which is used to address the distribution of electrons on the GaAs surface. Additionally, the prediction of the structural changes and binding energy trends through BOP function matches experimental observations well $[42,43]$.

$$
\begin{aligned}
E= & 1 / 2 \sum_{i=1}^{N} \sum_{j=i_{1}}^{i_{N}} \varphi_{i j}\left(r_{i j}\right)-\sum_{i=1}^{N} \sum_{j=i_{1}}^{i_{N}} \beta_{\sigma, i j}\left(r_{i j}\right) \cdot \theta_{(\sigma, i j)} \\
& -\sum_{i=1}^{N} \sum_{j=i_{1}}^{i_{N}} \beta_{(\pi, i j)}\left(r_{i j}\right) \cdot \theta_{(\pi, i j)}+U_{\text {prom }}
\end{aligned}
$$

where the $r_{i j}$ is the distance function for interatomic $i$ and $j \varphi_{i j}\left(r_{i j}\right)$ is the repulsive energy and short-range two-body function, $\beta_{\sigma, i j}\left(r_{i j}\right)$ and $\beta_{\pi, i j}\left(r_{i j}\right)$ present bond integrals, $\theta_{\sigma, i j}$ and $\theta_{\pi, i j}$ refer to specific bond-orders, $U_{\text {prom }}$ determines the promotion energy about sp-valent systems.

As Ziegler-Biersack-Littmark (ZBL) potential function [44] is suitable to calculate high-energy collisions accurately among $\mathrm{C}, \mathrm{Ga}$, and As atoms, it is employed to describe the occurrence of diamond cutting tool collision with GaAs workpiece edge, as follows in Eqs. (2-3).

$E_{i j}^{\mathrm{ZBL}}=\frac{1}{4 \pi \epsilon_{0}} \frac{Z_{i} Z_{j} e^{2}}{r_{i j}} \varphi\left(r_{i j} / a\right)+S\left(r_{i j}\right)$ 
Fig. 1 Orthogonal MD simulation model of SPDT on GaAs (lllll $\left.\begin{array}{lll}0 & 1\end{array}\right)$ surface along the [ $\left[\begin{array}{lll}1 & 1 & 0\end{array}\right]$ direction
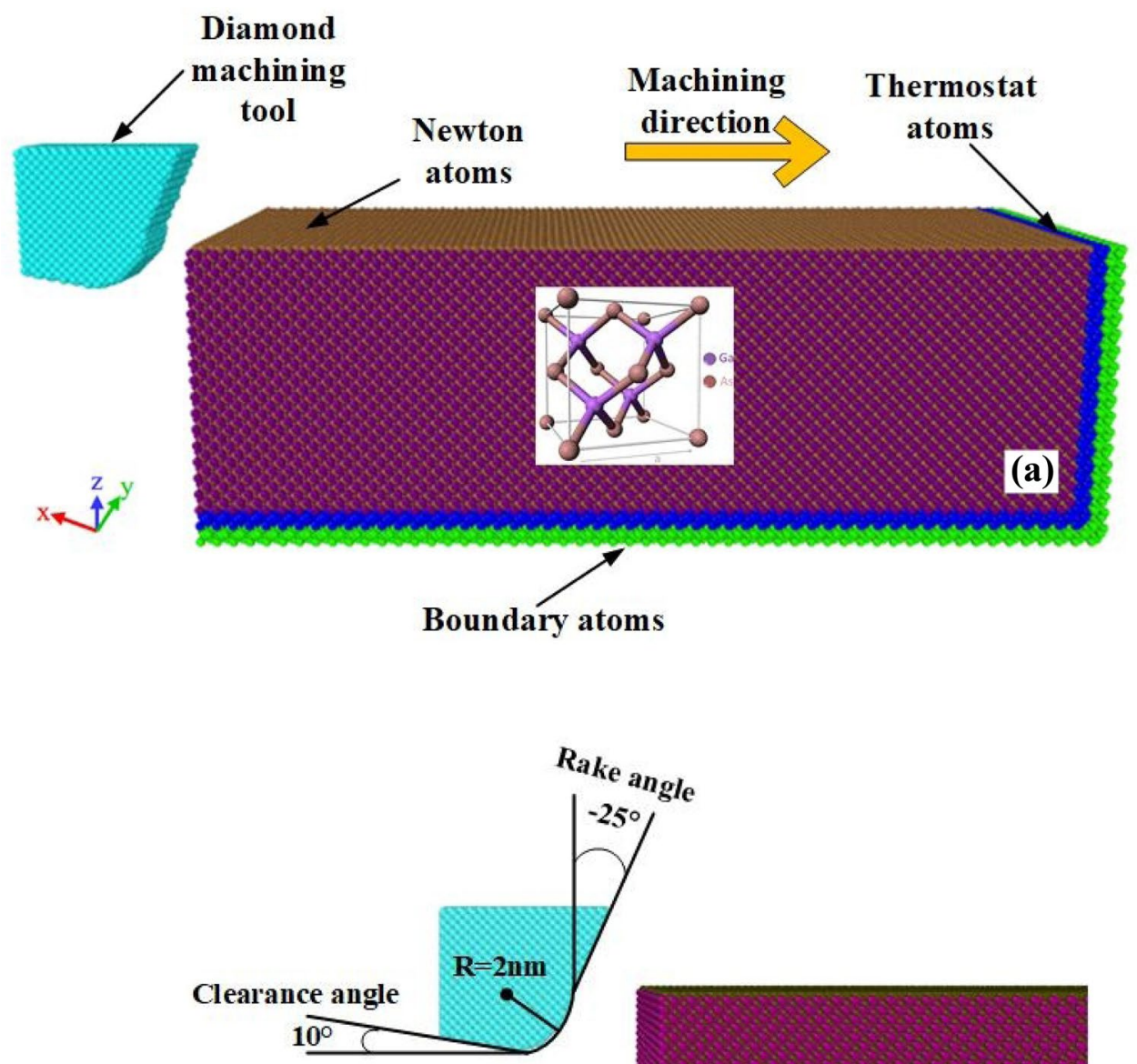

$a=\frac{0.46850}{Z_{i}^{0.23}+Z_{j}^{0.23}}$

where $\epsilon_{0}$ is the electrical permittivity of vacuum, $e$ is the electron charge, $Z_{i}$ and $Z_{j}$ are the nuclear charges of the two types atom, $r_{i j}$ is identical to mentioned above, $S\left(r_{i j}\right)$ refers to a switching function.

\subsection{Experimental Setup}

In order to verify the MD simulation results, SPDT of GaAs was carried out on an ultra-precision diamond turning machine equipped with air bearing slides and spindle. The experiment setup is shown in Fig. 2. The air bearing spindle has an axial motional error of less than $50 \mathrm{~nm}$. A single-crystal GaAs wafer (diameter of $50 \mathrm{~mm}$, thickness of $5 \mathrm{~mm}$ ) was glued on a copper fixture which was mounted on the vacuum chuck. The cutting direction was along the [ $\left[\begin{array}{lll}1 & 1 & 0\end{array}\right]$ direction on the (lllll) surface. A cubic lattice structure diamond turning tool with a rake angle of -25 degrees and a clearance angle of 10 degrees, which is consistent with the tool geometry used in MD simulation was selected to conduct diamond turning trial. The depth of cut and cutting speed were set as $10 \mu \mathrm{m}$ and $1.84 \mathrm{~m} / \mathrm{s}$, respectively. The detailed experimental parameters are shown in Table 2. The cutting force was measured by a dynamometer (Kistler 9129). Finally, the machined GaAs workpiece surface was measured by a white light interferometer (Zygo CP300) after ultrasonic cleaning in a 95 vol\% medical alcohol bath. The machining trials were performed on the (ll 001 ) surface of the GaAs wafer under a dry cutting condition and at a room temperature of 20 degrees in accordance with the MD simulation setup. 
Table 1 MD simulation parameters for SPDT of GaAs

\begin{tabular}{ll}
\hline GaAs lattice structure & Zinc-blende \\
\hline GaAs lattice constant & $5.65 \AA$ \\
Atom numbers of GaAs substrate & 190,080 \\
Substrate dimensions & $21 \mathrm{~nm} \times 10 \mathrm{~nm} \times 13.4 \mathrm{~nm}$ \\
Machining tool & Cubic diamond \\
Diamond lattice constant & $3.57 \AA$ \\
Atom numbers of the machining tool & 12,085 \\
Machining tool dimensions & $5.8 \mathrm{~nm} \times 5.0 \mathrm{~nm} \times 2.8 \mathrm{~nm}$ \\
Rake angle & $-25^{\circ}$ \\
Clearance angle & $10^{\circ}$ \\
Tool nose radius & $2 \mathrm{~nm}$ \\
Machining depth & $2 \mathrm{~nm}$ \\
Machining distance & $16 \mathrm{~nm}$ \\
Machining velocity & $200 \mathrm{~m} / \mathrm{s}$ \\
Machining lattice plane & $(001),(110),(111)$ \\
Machining direction & {$[110]$} \\
Equilibration temperature & $300 \mathrm{~K}$ \\
Potential functions & Tersoff, BOP, ZBL \\
Timestep & $1 \mathrm{fs}$ \\
\hline
\end{tabular}

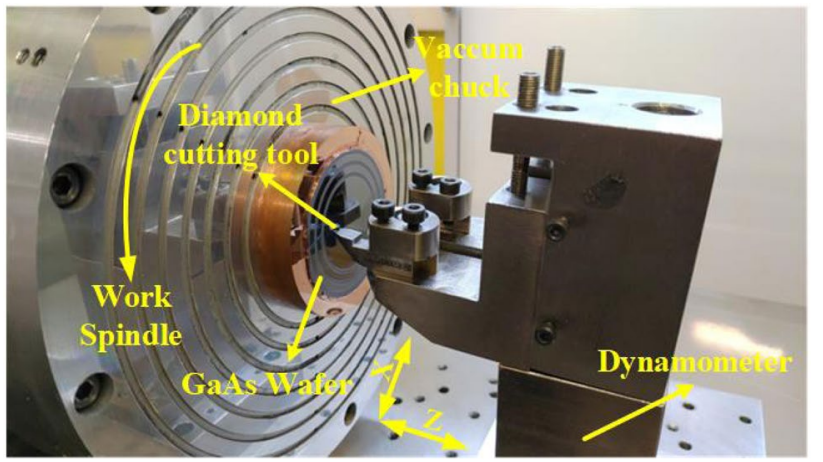

Fig. 2 Experimental setup of single-point diamond turning of GaAs

Table 2 Diamond turning parameters

\begin{tabular}{lll}
\hline Parameters & Unit & Values \\
\hline Cutting depth & $\mu \mathrm{m}$ & 0.3 \\
Cutting speed & $\mathrm{m} / \mathrm{s}$ & 1.84 \\
Feed rate & $\mu \mathrm{m} / \mathrm{rev}$ & $0.5,1.0,1.5$ \\
Tool nose radius & $\mathrm{mm}$ & 5 \\
Tool cutting edge radius & $\mathrm{nm}$ & 73.79 \\
Tool rake angle & $\circ$ & -25 \\
Tool clearance angle & $\circ$ & 10 \\
\hline
\end{tabular}
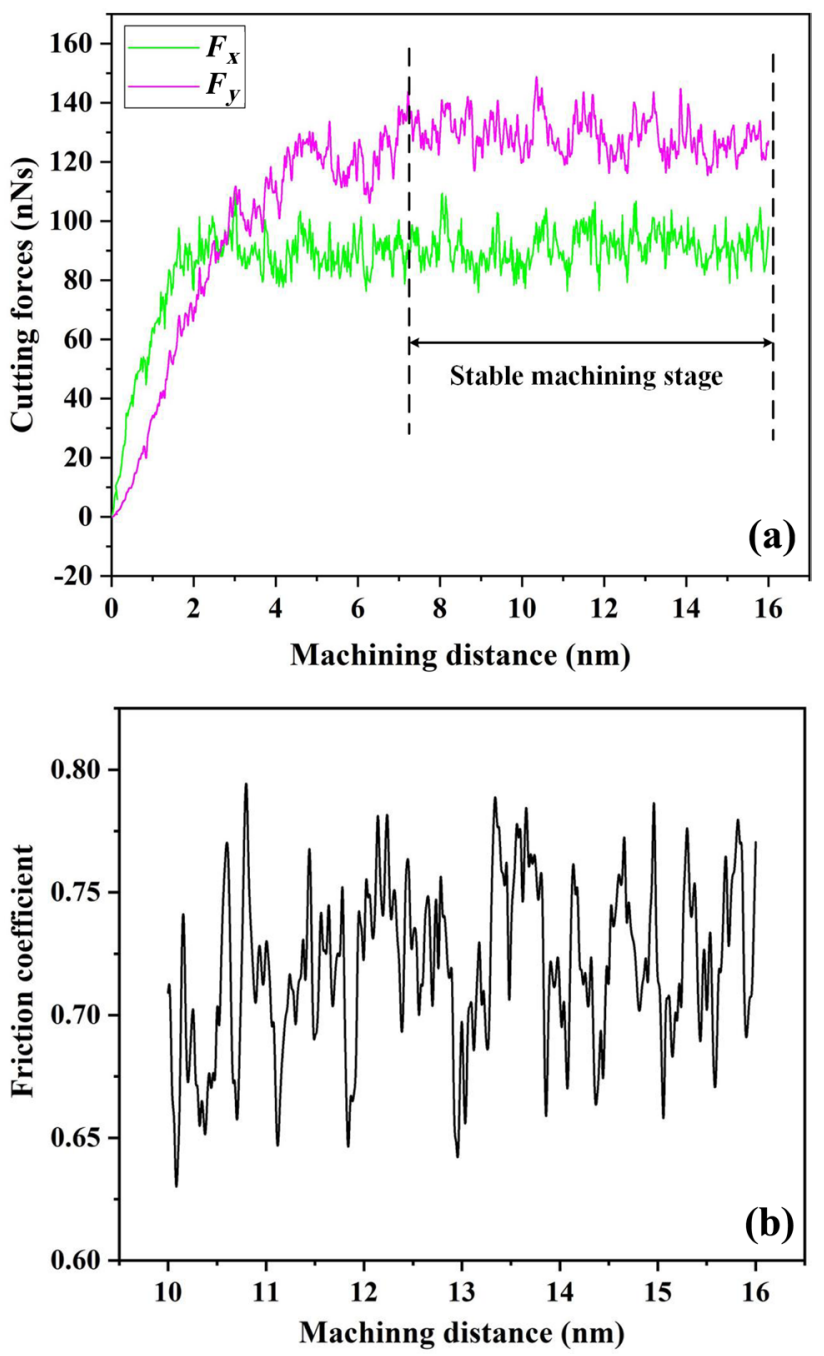

Fig. 3 a Variation of the cutting forces in the $X$ and $Y$ directions against cutting distances. b Variation of friction coefficient of MD simulation results during stable machining stage

\section{Results and Discussion}

\subsection{Cutting Forces and Experimental Validation}

The cutting force is a pivotal parameter for the study of the SPDT process, as it is directly related to the formation of cutting chip. Figure 3 a shows the variation of the cutting forces in the $X$ and $Y$ directions during the cutting process. The tangential cutting force $F_{x}$ rose steeply in the first $2 \mathrm{~nm}$ of cutting and then fluctuated around $90 \mathrm{nNs}$ at the following $14 \mathrm{~nm}$ of cutting with a peak force of $109.373 \mathrm{nNs}$. The normal force $F_{y}$ increased dramatically in the first $7 \mathrm{~nm}$ of cutting and also slightly fluctuated at approximately $130 \mathrm{nNs}$ with a maximum value of $149.33 \mathrm{nNs}$. These data indicate that the cutting chip was generated at the initial $7 \mathrm{~nm}$ of cutting and then stabilized in the following $9 \mathrm{~nm}$ of cutting. 
Fig. 4 Variations of normal force $F_{y}$ at the feed rates of 0.5 , 1.0 , and $1.5 \mu \mathrm{m} / \mathrm{rev}$, respectively

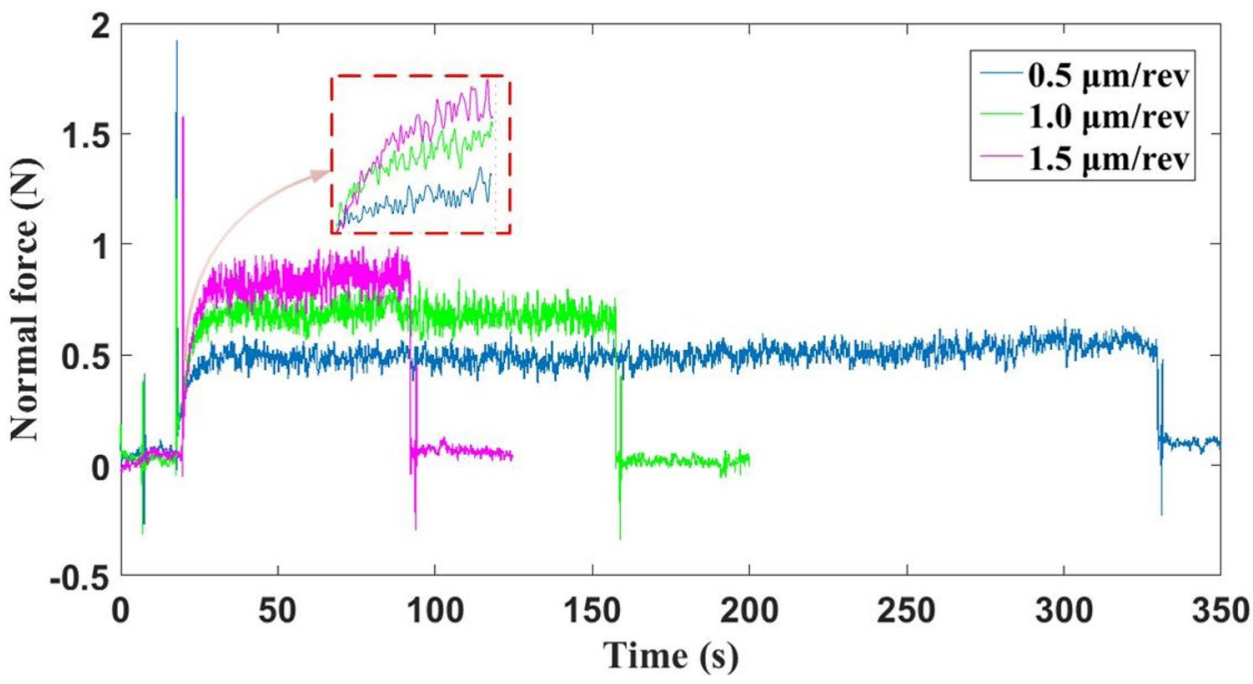

During the fluctuation of cutting force, GaAs's perfect zincblende phase structure was squeezed and deformed by the diamond cutting tool and then the lattice recombined to evolve to a new phase, which will be presented in the following analysis of variation of coordination number $(\mathrm{CN})$ section. In addition, as shown in Fig. 3a, the growth rate of the normal force along the $Y$ direction is less than that of the tangential force along the $X$ direction during the chip formation stage but it then became larger than that of the tangential force. This phenomenon is attributed to the use of a diamond cutting tool with a large negative rake angle, which provides a normal cutting force larger than the tangential cutting force. Additionally, the average friction coefficient $\left(F_{x} / F_{y}\right)$ was calculated to be 0.719 during the stable machining stage from the cutting distances of $10-16 \mathrm{~nm}$ and its variation is shown in Fig. $3 \mathrm{~b}$.

The SPDT of GaAs workpiece experiments were carried out under different feed rates $(0.5,1.0$, and $1.5 \mu \mathrm{m} /$ rev). Firstly, the normal force was extracted for each case, as indicated in Fig. 4. It suggests that the normal force of the machining tool experiences a rapid increase in the first $20 \mathrm{~s}$ and keeps steady fluctuation after $40 \mathrm{~s}$, which is responsible for the achievement of a stable chip. Consequently, it is obvious to see that the variation trend of experimental normal force in Fig. 4 is consistent with that of MD normal force simulation in Fig. 3a. Hence, the MD simulation results were verified qualitatively to some extent.

\subsection{GaAs Material Ductile Response}

Ductile response of single-crystal GaAs was observed from the MD simulation results, as shown in Fig. 7a, where no cracks or brittle fracture were observed. To reveal the underlying reason for the material ductile response, the average values of the hydrostatic stress at the cutting zone were calculated and are shown in Fig. 5. The hydrostatic stress

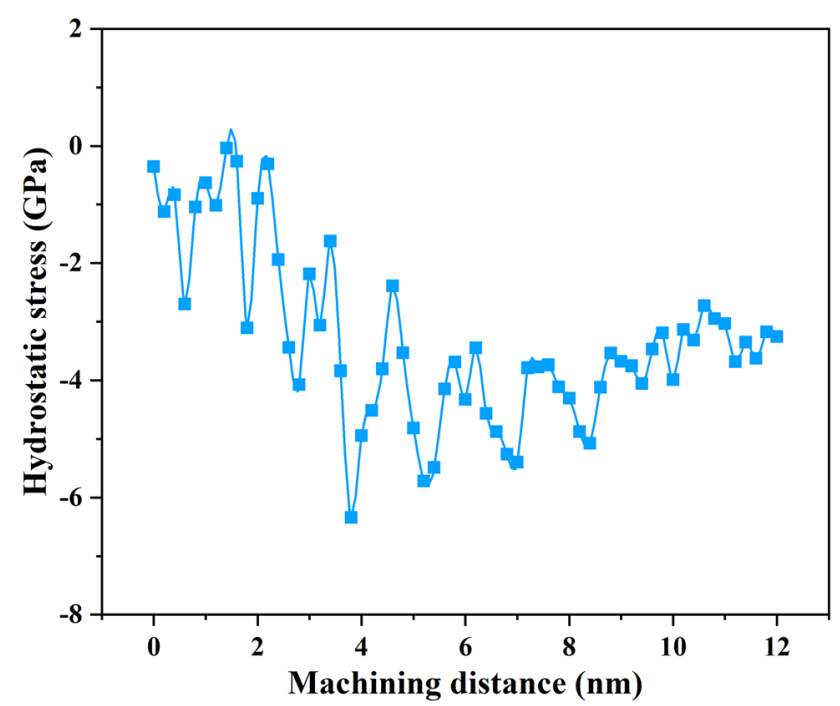

Fig. 5 Variation of hydrostatic stress value during SPDT

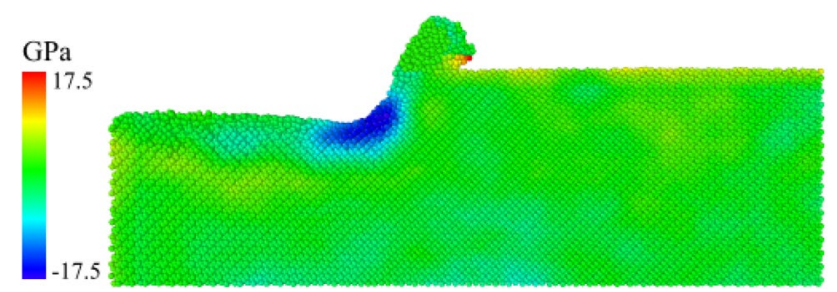

Fig. 6 Hydrostatic stress distribution of the machined GaAs substrate at a cutting distance of $12 \mathrm{~nm}$

firstly increased rapidly and then fluctuated around $4 \mathrm{GPa}$ during the SPDT process. Additionally, the hydrostatic stress distribution within the machined GaAs substrate, as shown in Fig. 6, revealed that the maximum hydrostatic stress in the cutting zone took place underneath the cutting tool and could rise up to $17.5 \mathrm{GPa}$. Phase transformation 

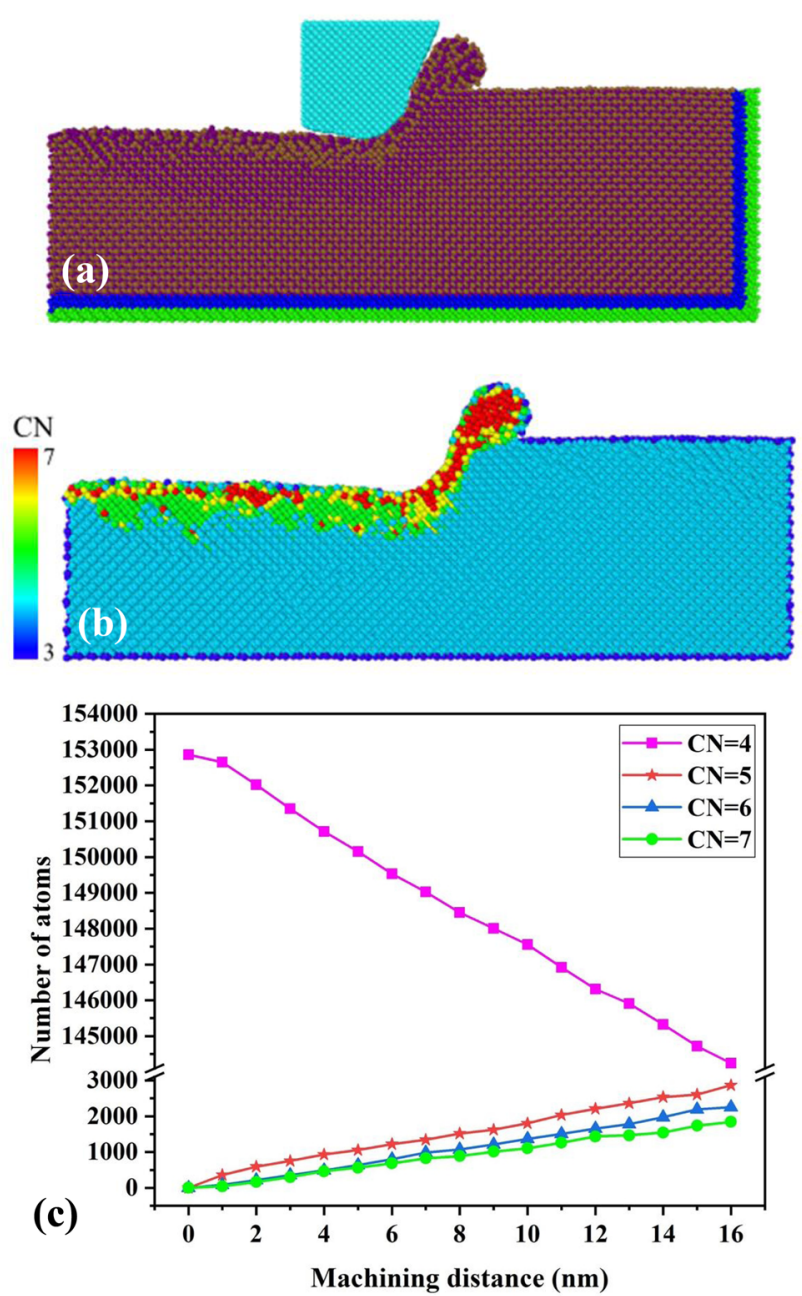

Fig. 7 a Snapshot of MD simulation at cutting distance of $16 \mathrm{~nm}$. b Cross-sectional image of $\mathrm{CN}$ distribution at the cutting distances of $16 \mathrm{~nm}$. $\mathbf{c}$ Evolution of various $\mathrm{CN}$ atoms against machining distance

of single-crystal GaAs under the same scale of hydrostatic stress was observed in Shigeaki et al.'s experimental work [45]. Therefore, the extremely high hydrostatic stress value during SPDT implies the occurrence of phase transformation of single-crystal GaAs.

To further confirm the occurrence of phase transition of single-crystal GaAs under high hydrostatic stress during SPDT, the coordination number $(\mathrm{CN})$, i.e., the number of the nearest-neighbor atoms around a central atom in a compound, was calculated to characterize the phase transformation of GaAs. The threefold-coordinated value ( $\mathrm{CN}$ of 3) is indicative of dangling bonds on the surface of $\mathrm{GaAs}$ and the fourfold-coordinated value ( $\mathrm{CN}$ of 4$)$ refers to the intrinsic cubic zinc-blende structure (GaAs-I). The sixfold-coordinated value ( $\mathrm{CN}$ of 6 ) presents the rock-salt structure (GaAs-II) and the fivefold-coordinated value $(\mathrm{CN}$ of 5) atoms appears the intermediate status between intrinsic zinc blende structure (GaAs-I) and rock-salt structure
(GaAs-II), followed by the amorphous structure of sevenfold-coordinated value ( $\mathrm{CN}$ of 7 ) atoms. Figure $7 \mathrm{~b}$ shows that the machined deformation zone is largely comprised of two kinds of lattice structures: green color atoms with intermediate status CN 5 and yellow color atoms with rocksalt structure (GaAs-II) CN 6, which is attributed to the slow evolution of amorphous structure of the atoms of $\mathrm{CN}$ 7 located at the cutting chip. Additionally, the amorphous structure constituted the main part of the cutting chip. The study of temperature distribution among GaAs workpiece during nanomachining process showed that the maximum temperature appeared at the top of the cutting chip, which indicates that the amorphous lattice structure possessed very high lattice deformation energy. As can be seen from Fig. 7c, the number of atoms of $\mathrm{CN}$ of four significantly declined, while the atoms with $\mathrm{CN}$ of five, $\mathrm{CN}$ of six, and $\mathrm{CN}$ of seven increased rapidly with the increasing cutting distance. The $\mathrm{CN}$ change from four to six implies that the lattice structure of the single-crystal GaAs was transformed from cubic zinc-blende structure to rock-salt structure [46]. In other words, the phase of the single-crystal GaAs was changed from GaAs-I to GaAs-II. Therefore, the reason of the material ductile response of single-crystal GaAs during SPDT is the occurrence of phase transformation of $\mathrm{GaAs}$ under extremely high stress, i.e., the so-called high-pressure phase transformation (HPPT) took place. Additionally, neither dislocation nor twinning was observed in this MD simulation study. By contrast, various sorts of dislocations were found out in the MD simulation of diamond turning of $\mathrm{Si}$ and $\mathrm{SiC}$ $[47,48]$. The authors would like to undertake and report a comparison study in the future to reveal the underlying reasons to cause the difference in MD simulation results although they are different brittle materials.

\subsection{Temperature Distribution During SPDT}

Rise of cutting temperature can cause thermal deformation or damage to the workpiece, therefore the cutting temperature is a significant influential factor on the machined surface form accuracy and integrity. In this study, the cutting temperature is calculated by using the following equation,

$T=\frac{2 \mathrm{~K} \cdot \mathrm{E} .}{3 N K_{\mathrm{b}}}$

where the K.E. refers to the kinetic energy of GaAs substrate atoms, $N$ is the atoms quantity, $K_{\mathrm{b}}$ represents the Boltzmann constant. The temperature was averaged over a cubic zone with a length of $10 \AA$. A defined elemental atomic volume $(16 \mathrm{~nm} \times 2 \mathrm{~nm} \times 3 \mathrm{~nm})$ was set to the primary cutting zone. A dramatic rise of cutting temperature with the increase of cutting distance was observed in the diagram of the variation of cutting temperature shown in 
Fig. 8 a Temperature distribution on the GaAs workpiece after $16 \mathrm{~nm}$ of cut. b Increase of temperature during SPDT machining
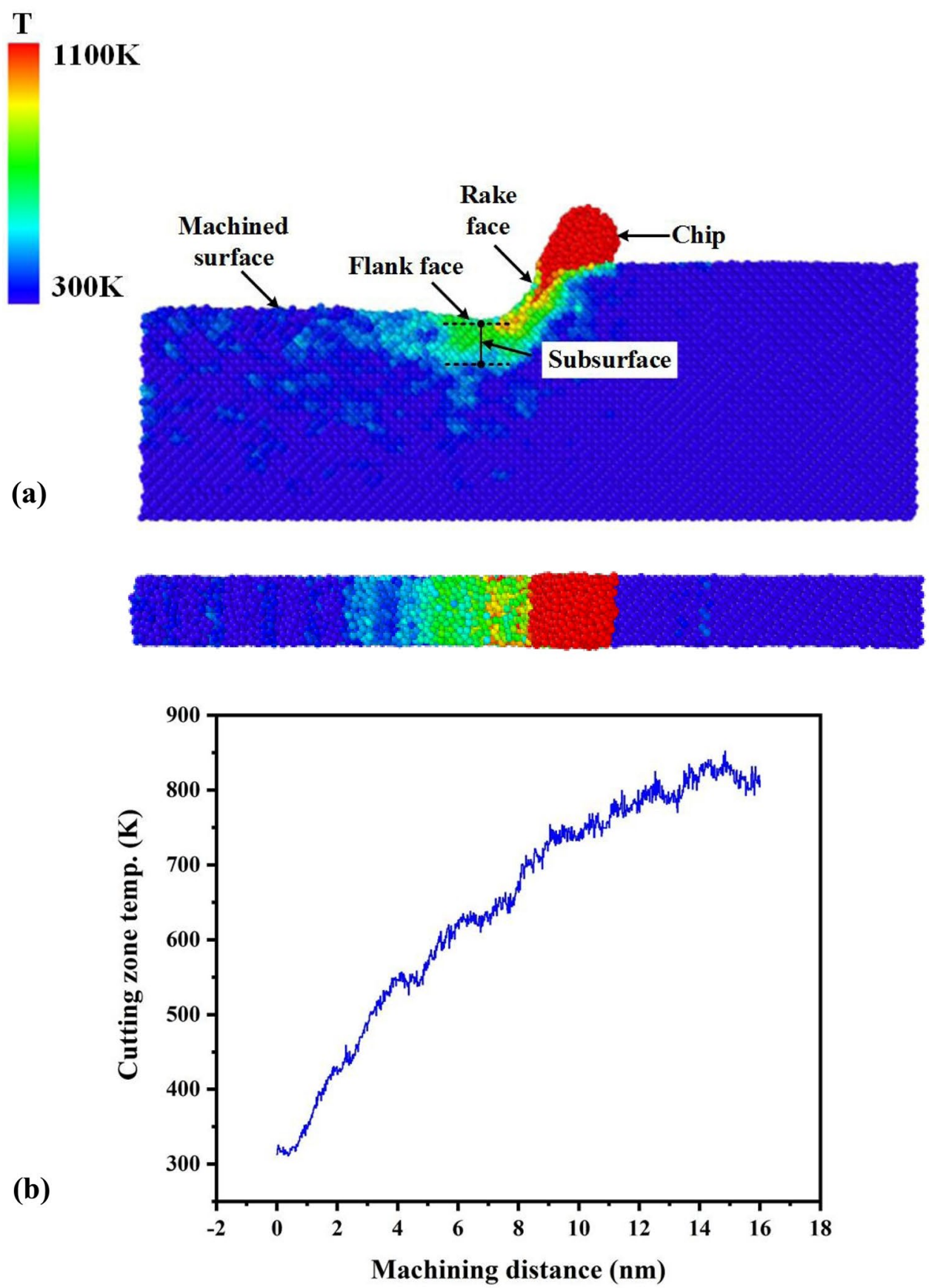

Fig. $8 \mathrm{~b}$. The cutting temperature reached a maximum value of $851.86 \mathrm{~K}$ after the cutting distance of $15 \mathrm{~nm}$. Figure 8a illustrates the temperature distribution using various color zones. The same phenomenon has been observed on other ductile and brittle materials such as $\mathrm{Cu}, \mathrm{Al}$, and $\mathrm{Si}$ materials $[40,41]$. According to temperature distribution, the highest temperature appeared at the top of the chip, which is up to $1100 \mathrm{~K}$. This is because the top of the chip experienced the most serious lattice deformation, which could possess the maximum transformation lattice energy. Furthermore, the diamond tool rake face and flank face also showed extremely high temperatures, nearly 900 and $700 \mathrm{~K}$, respectively, since the rake face pressed against the GaAs atoms and friction occurred between the flank face and the machined GaAs surface. Additionally, the subsurface of the machine GaAs at $2 \mathrm{~nm}$ underneath the flank face showed a high temperature of approximately $700 \mathrm{~K}$. Finally, most of the atoms beside the substrate edge could keep an almost stable temperature $300 \mathrm{~K}$, which benefited from the heat dissipation of the thermostat layer atoms. 


\subsection{The Influence of Crystalline Orientation}

This paper analyzes the effect of crystalline orientation on the cutting chip, sidewall morphology, and friction coefficient among typical crystal orientations, including the (0 0 1) surface along the $\left[\begin{array}{lll}1 & 1 & 0\end{array}\right]$ cutting direction, $\left(\begin{array}{lll}1 & 1 & 0\end{array}\right)$ surface along the $\left[\begin{array}{lll}0 & 0 & 1\end{array}\right]$ cutting direction, and $\left(\begin{array}{lll}1 & 1 & 1\end{array}\right)$ surface along

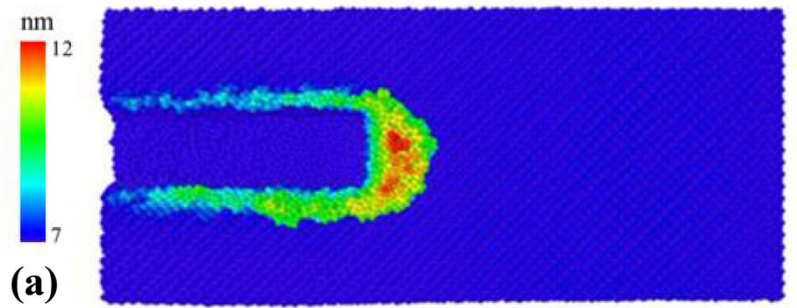

(b)

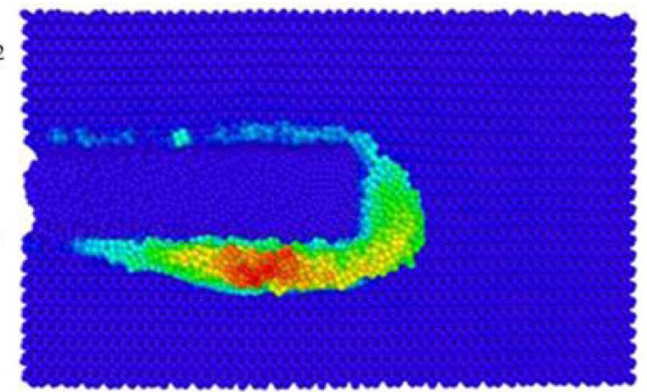

(c)

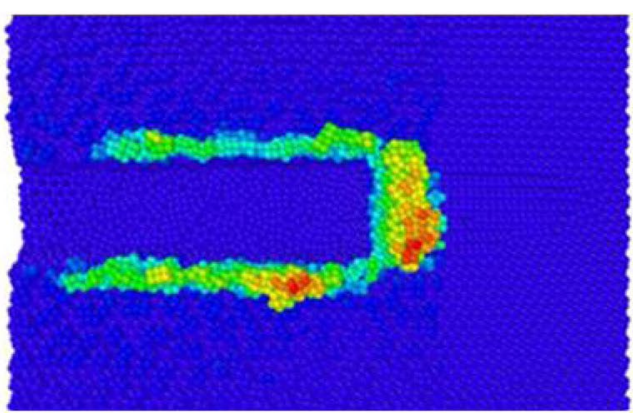

Fig. 9 The colored morphology of cutting chip and sidewall pile-up during along the $Y$ direction a GaAs ( $\left(\begin{array}{lll}0 & 0 & 1\end{array}\right)$, b GaAs ( $\left.\begin{array}{lll}1 & 1 & 0\end{array}\right)$, c GaAs (1 111 ) the $\left[\begin{array}{lll}1 & \overline{1} & 0\end{array}\right]$ cutting direction. According to Figs. 9 and 10, it is clear to see that the GaAs atoms piled up on both sides of the nanogroove when cutting the (lllll $\left.0 \begin{array}{lll}0 & 1\end{array}\right)$ and $\left(\begin{array}{lll}1 & 1 & 1\end{array}\right)$ surfaces. Furthermore, when cutting the $\left(\begin{array}{lll}1 & 1 & 1\end{array}\right)$ surface, the $\mathrm{GaAs}$ atoms were evenly distributed on both sides, while for cutting the $\left(\begin{array}{lll}0 & 0 & 1\end{array}\right)$ surface, more atoms were piled up on the right side than the left. Almost all the removed atoms were piled up on the right side for machining $\left(\begin{array}{lll}1 & 1 & 0\end{array}\right)$ surface. Meanwhile, the left-hand side atoms could have more atom pile-ups than the right-hand side when cutting along the opposite direction $\left[\begin{array}{lll}0 & 0 & \overline{1}\end{array}\right]$ on $\left(\begin{array}{lll}1 & 1 & 0\end{array}\right)$ surface, as shown in Fig. 10c. This occurrence might be attributed to different atomic density and inter-atomic distance in different $\mathrm{GaAs}$ crystal surfaces.

Additionally, the cutting forces and friction coefficients were calculated for three different kinds of crystal surfaces. The results are shown in Table 3. The minimum friction coefficient of 0.630 was found when cutting the $\left(\begin{array}{lll}1 & 1 & 1\end{array}\right)$ face along the $\left[\begin{array}{lll}1 & \overline{1} & 0\end{array}\right]$ direction, which means the SPDT process has the least resistance in this crystalline orientation and cutting direction combination. The maximum friction coefficient of 0.719 was found when cutting the GaAs $(0$ $\left.\begin{array}{ll}0 & 1\end{array}\right)$ surface along the $\left[\begin{array}{lll}1 & 1 & 0\end{array}\right]$ direction. Cutting the $\left(\begin{array}{lll}1 & 1 & 0\end{array}\right)$ surface along the $\left[\begin{array}{lll}0 & 0 & 1\end{array}\right]$ direction shows the intermediate friction coefficient of 0.680 . The results suggested that the $\left[\begin{array}{lll}1 & 1 & 0\end{array}\right]$ direction is the hardest machining direction on the $(0$ 01 ) surface. This finding is consistent with the conclusion drawn in the experimental study on circumferential distribution of material brittle fracture index of single-crystal GaAs by Chen et al. [31]. In theory, if the GaAs material showed ductile response on the difficult-to-cut surface and cutting

Table 3 The average value of tangential force, normal force, and friction coefficient during SPDT on different crystalline orientations

\begin{tabular}{llll}
\hline & $F x(\mathrm{nNs})$ & $F y(\mathrm{nNs})$ & $\mathrm{FC}$ \\
\hline$\left(\begin{array}{lll}0 & 0 & 1\end{array}\right)$ & 91.56 & 127.35 & 0.719 \\
$\left(\begin{array}{lll}1 & 1 & 0\end{array}\right)$ & 82.27 & 120.99 & 0.680 \\
$\left(\begin{array}{lll}1 & 1 & 1\end{array}\right)$ & 66.35 & 105.31 & 0.630 \\
\hline
\end{tabular}
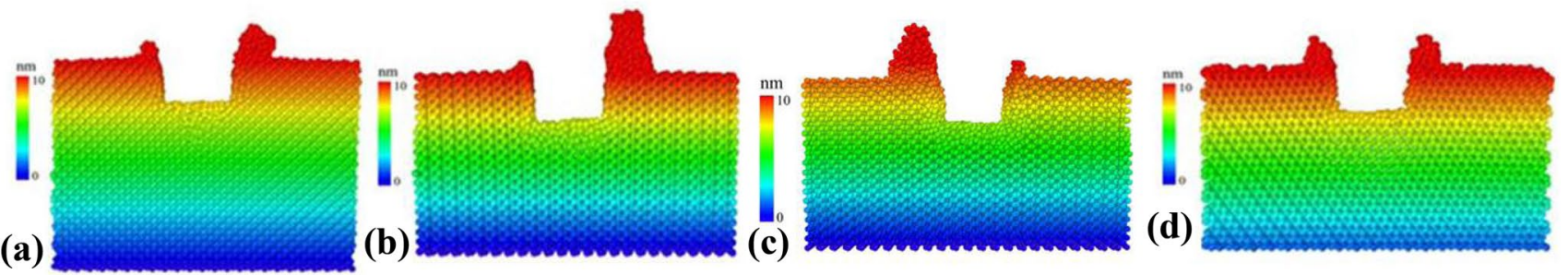

Fig. 10 The pile-up of the sidewall during SPDT along the $Y$ direction a GaAs $\left(\begin{array}{lll}0 & 0 & 1\end{array}\right), \mathbf{b}$ GaAs $\left(\begin{array}{lll}1 & 1 & 0\end{array}\right)$ along $\left[\begin{array}{lllll}0 & 0 & 1\end{array}\right], \mathbf{c}$ GaAs $\left(\begin{array}{llll}1 & 1 & 0\end{array}\right)$ along $\left[\begin{array}{lll}0 & 0 & \overline{1}\end{array}\right]$ d GaAs ( $\left(\begin{array}{lll}1 & 1 & 1\end{array}\right)$ 
Fig. 11 Measured machined surface of GaAs in the SPDT experiment direction, brittle fracture-free machined surfaces could therefore be achieved in other crystal orientations and cutting direction under the same machining parameters. In this study, we selected the most difficult-to-machine $\left(\begin{array}{lll}0 & 0 & 1\end{array}\right)$ surface to carry out SPDT experiments along the most difficultto-machine cutting direction $\left[\begin{array}{lll}1 & 1 & 0\end{array}\right]$. The experimental result showed a nano-smooth machined GaAs surface finish with a surface roughness Ra of $8 \mathrm{~nm}$ could be obtained when using a feed rate of $0.5 \mu \mathrm{m} / \mathrm{rev}$, a spindle speed of $800 \mathrm{rpm}$, and a depth of cut $0.3 \mu \mathrm{m}$, which is shown in Fig. 11.

\section{Conclusions}

In this work, both MD simulations and SPDT experiments have been employed to investigate atomistic aspects of ductile response of single-crystal GaAs material. The conclusions can be drawn as follows:

1. The SPDT is accompanied by a sharp rise in temperature in the cutting zone. The generated highest temperature is located at the cutting chip, in which the temperature can reach up to $1100 \mathrm{~K}$. Furthermore, the rake face and flank face also have extremely high temperatures of nearly 900 and $700 \mathrm{~K}$, respectively.
2. The underlying reason for the single-crystal GaAs ductile response during SPDT process is the high-pressure phase transformation (HPPT), in which the intrinsic zinc-blende phase structure GaAs-I of single-crystal GaAs transformed to a rock-salt phase structure GaAsII under high hydrostatic stress. In addition, the intermediate phase structure and rock-salt phase structure dominate the machined deformation zone while the amorphization structure mainly consists of the formed chip.

3. Single-crystal GaAs shows the strong anisotropic machinability during SPDT. When cutting the $\left(\begin{array}{lll}0 & 0 & 1\end{array}\right)$ surface along the $\left[\begin{array}{lll}1 & 1 & 0\end{array}\right]$ direction, the simulated morphology shows that more atoms pile up on the right side than the left side, while GaAs atoms are well distributed on both sides when cutting the $\left(\begin{array}{lll}1 & 1 & 1\end{array}\right)$ surface along the $\left[\begin{array}{lll}1 & 1 & 0\end{array}\right]$ direction. With regards to cutting the $\left(\begin{array}{lll}1 & 1 & 0\end{array}\right)$ surface along the $\left[\begin{array}{lll}0 & 0 & 1\end{array}\right]$ direction, almost all piled-up GaAs atoms appeared on the right side. The maximum friction coefficient is found when cutting the GaAs (0 0 1) surface along the $\left[\begin{array}{lll}1 & 1 & 0\end{array}\right]$ direction while cutting the (1 $\left.\begin{array}{lll}1 & 1 & 1\end{array}\right)$ surface along the $\left[\begin{array}{lll}1 & \overline{1} & 0\end{array}\right]$ direction possessed the minimum friction coefficient. The friction coefficient when cutting the $\left(\begin{array}{lll}1 & 1 & 0\end{array}\right)$ surface along the $\left[\begin{array}{lll}0 & 0 & 1\end{array}\right]$ direction is intermediate among these three typical crystalline orientations and cutting-direction combinations. 
Acknowledgements The authors would like to thank EPSRC (EP/ K018345/1 and EP/T024844/1) and the Royal Society-NSFC international exchange programme (IEC\NSFC \181474) for providing financial support for this research. The authors also acknowledge the use of the EPSRC (EP/K000586/1) funded ARCHIE-WeSt High-Performance Computer at the University of Strathclyde for the MD simulation study.

Data Availability All data underpinning this publication are openly available from the University of Strathclyde KnowledgeBase at https:// doi.org/10.15129/746765d8-0cd3-4320-ac8e-fe4d3192e90a.

Open Access This article is licensed under a Creative Commons Attribution 4.0 International License, which permits use, sharing, adaptation, distribution and reproduction in any medium or format, as long as you give appropriate credit to the original author(s) and the source, provide a link to the Creative Commons licence, and indicate if changes were made. The images or other third party material in this article are included in the article's Creative Commons licence, unless indicated otherwise in a credit line to the material. If material is not included in the article's Creative Commons licence and your intended use is not permitted by statutory regulation or exceeds the permitted use, you will need to obtain permission directly from the copyright holder. To view a copy of this licence, visit http://creativecommons.org/licenses/by/4.0/.

\section{References}

1. Blankemeier L, Rezaeifar F, Garg A, Kapadia R (2019) Integrated photonics for low transverse emittance, ultrafast negative electron affinity GaAs photoemitters. J Appl Phys 126(3):033102

2. Nanayakkara TR et al (2018) Electron heating induced by microwave photoexcitation in the GaAs/AlGaAs two-dimensional electron system. Phys Rev B 98(3):1-6

3. Kunets VP et al (2008) Low thermal drift in highly sensitive doped channel Al 0.3 Ga 0.7As/GaAs/In 0.2Ga 0.8As micro-Hall element. J Mater Sci Mater Electron 19(8-9):776-782

4. Mazouz H, Logerais PO, Belghachi A, Riou O, Delaleux F, Durastanti JF (2015) Effect of electron irradiation fluence on the output parameters of GaAs solar cell. Int J Hydrog Energy 40(39):13857-13865

5. Blin S et al (2013) Wireless communication at $310 \mathrm{GHz}$ using GaAs high-electron-mobility transistors for detection. J Commun Netw 15(6):559-568

6. Li HO et al (2015) Conditional rotation of two strongly coupled semiconductor charge qubits. Nat Commun 6:1-9

7. Cao G et al (2013) Ultrafast universal quantum control of a quantum-dot charge qubit using Landau-Zener-Stückelberg interference. Nat Commun 4:1-7

8. Shi Z et al (2014) Fast coherent manipulation of three-electron states in a double quantum dot. Nat Commun 5:1-5

9. Cheng W, Hu CL, Jiao GC, Miao Z, Zhang N (2013) Analyses the character of GaAs photocathode by diffraction curve from spectrophotometer. Key Eng Mater 552:287-291

10. Kudrawiec R, Misiewicz J, Fisher M, Forchel A (2004) Optical properties of GaInNas/GaAs quantum wells: character of optical transitions and carrier localisation effect. Phys Status Solidi Appl Res 201(2):364-367

11. Mäder KA, Wang LW, Zunger A (1995) Electronic consequences of random layer-thickness fluctuations in AlAs/GaAs superlattices. J Appl Phys 78(11):6639-6657
12. Gangopadhyay A, Maros A, Faleev N, Smith DJ (2019) Strain relaxation in low-mismatched $\mathrm{GaAs} / \mathrm{GaAs}_{1-\mathrm{x}} \mathrm{Sb}_{\mathrm{x}} / \mathrm{GaAs}$ heterostructures. Acta Mater 162:103-115

13. Ahn HJ, Il Chang W, Kim SM, Park BJ, Yook JM, Eo YS (2019) $28 \mathrm{GHz}$ GaAs pHEMT MMICs and RF front-end module for $5 \mathrm{G}$ communication systems. Microw Opt Technol Lett 61(4):878-882

14. Nitesh RS, Rajendran J, Ramiah H, Abd sssssManaf A (2018) A $700 \mathrm{MHz}$ to $2.5 \mathrm{GHz}$ cascode $\mathrm{GaAs}$ power amplifier for multi-band pico-cell achieving $20 \mathrm{~dB}$ gain, $40 \mathrm{dBm}$ to $45 \mathrm{dBm}$ OIP3 and $66 \%$ peak PAE. IEEE Access 6:818-829

15. Florencio Díaz R, Rodríguez Boix R, Carrasco Yépez F, Encinar Garcinuño J, Barba Gea M, Pérez Palomino G (2014) Broadband reflect arrays made of cells with three coplanar parallel dipoles. Microw Opt Technol Lett 56(3):748-753

16. Sasani K, Abbasi SP, Sabrlouy Kolian B, Zabihi MS, Sabbaghzadeh J (2011) Relationship between concentration, time and surface roughness of GaAs wafer in lapping process: an experimental investigation. In: 2011 symposium on photonics and optoelectronics, SOPO 2011, pp 1-4

17. Prakash SJ, Tyagi R, Gupta A (2011) Backside thinning of GaAs wafer by lapping using DOE approach. In: India international conference on power electronics, IICPE 2010, pp 1-4

18. Mcmeekin SG, Robertson M, Mcgheeb L, Winfield JM (1992) Chemimechanical polishing of gallium arsenide to subnanometre surface finish. J Mater Chem 2(3):367-368

19. Wu YH, Chang L (2010) Chemical polishing method of GaAs specimens for transmission electron microscopy. Micron 41(1):20-25

20. Antwi EK, Liu K, Wang H (2018) A review on ductile mode cutting of brittle materials. Front Mech Eng 13(2):251-263

21. Venkatesh VC, Inasaki I, Toenshof HK, Nakagawa T, Marinescu ID (1995) Observations on polishing and ultraprecision machining of semiconductor substrate materials. CIRP Ann Manuf Technol 44(2):611-618

22. Ma Y, Ballesteros G, Zajac JM, Sun J, Gerardot BD (2015) Highly directional emission from a quantum emitter embedded in a hemispherical cavity. Opt Lett 40(10):2373

23. Blake PN, Scattergood RO (1990) Ductile-regime machining of germanium and silicon. J Am Ceram Soc 73(4):949-957

24. Li Z, Zhang X (2017) Subsurface deformation of germanium in ultra-precision cutting: characterization of micro-Raman spectroscopy. Int J Adv Manuf Technol 91(1-4):213-225

25. Goel S, Luo X, Reuben RL (2013) Wear mechanism of diamond tools against single-crystal silicon in single-point diamond turning process. Tribol Int 57:272-281

26. Goel S, Luo X, Comley P, Reuben RL, Cox A (2013) Brittleductile transition during diamond turning of single-crystal silicon carbide. Int J Mach Tools Manuf 65:15-21

27. Mir A, Luo X, Cheng K, Cox A (2018) Investigation of influence of tool rake angle in single-point diamond turning of silicon. Int J Adv Manuf Technol 94(5-8):2343-2355

28. Jasinevicius RG (2006) Influence of cutting conditions scaling in the machining of semiconductors crystals with single-point diamond tool. J Mater Process Technol 179(1-3):111-116

29. Fang ZJYFZ (1999) Ultra-precision cutting for gallium arsenide. In: Proceedings of the ASPE annual meeting, pp 62-66

30. Wang S, Pirouz P (2007) Mechanical properties of undoped GaAs. II: the brittle-to-ductile transition temperature. Acta Mater 55(16):5515-5525

31. Chen J, Luo X, Ding F, Rao X, Zhang J (2020) Fundamental study of diamond turning of single-crystal gallium arsenide. Precis Eng 62:71-82 
32. Goel S, Luo X, Agrawal A, Reuben RL (2015) Diamond machining of silicon: a review of advances in molecular dynamics simulation. Int J Mach Tools Manuf 88:131-164

33. Goel S, Luo X, Reuben RL, Bin Rashid W (2011) Atomistic aspects of ductile responses of cubic silicon carbide during nanometric cutting. Nanoscale Res Lett 6:1-9

34. Xiao G, To S, Zhang G (2015) The mechanism of ductile deformation in ductile regime machining of $6 \mathrm{H} \mathrm{SiC}$. Comput Mater Sci 98:178-188

35. Dai H, Chen G, Fang Q, Yin J (2016) The effect of tool geometry on subsurface damage and material removal in nanometric cutting single-crystal silicon by a molecular dynamics simulation. Appl Phys A Mater Sci Process 122(9):1-16

36. Chavoshi SZ, Goel S, Luo X (2015) Molecular dynamics simulation investigation on the plastic flow behaviour of silicon during nanometric cutting. Model Simul Mater Sci Eng 24(1):015002

37. Yi D, Li J, Zhu P (2018) Study of nanoscratching process of GaAs using molecular dynamics. Crystals 8(8):321

38. Plimpton SJ (1995) Fast parallel algorithms for short range molecular dynamics. J Comput Phys 117:1-19

39. Stukowski A (2010) Visualization and analysis of atomistic simulation data with OVITO-the open visualization tool. Model Simul Mater Sci Eng 18(1):015012

40. Nose $S$ (1984) A unified formulation of the constant temperature molecular-dynamics methods. J Chem Phys 81:511-519

41. Tersoff J (1989) Modeling solid-state chemistry: interatomic potentials for multicomponent systems. Phys Rev B 39(8):5566-5568

42. Ward DK, Zhou XW, Wong BM, Doty FP, Zimmerman JA (2012) Analytical bond-order potential for the cadmium telluride binary system. Phys Rev B Condens Matter Mater Phys 85(11):1-19

43. Pettifor DG, Oleinik II (2002) Analytic bond-order potential for open and close-packed phases. Phys Rev B Condens Matter Mater Phys 65(17):1-4

44. Ziegler JF, Ziegler MD, Biersack JP (2010) SRIM: the stopping and range of ions in matter (2010). Nucl Instrum Methods Phys Res Sect B Beam Interact Mater Atoms 268(11-12):1818-1823

45. Ono S, Kikegawa T (2018) Phase transformation of GaAs at high pressures and temperatures. J Phys Chem Solids 113:1-4

46. Besson JM, Itié JP, Polian A, Weill G, Mansot JL, Gonzalez J (1991) High-pressure phase transition and phase diagram of gallium arsenide. Phys Rev B 44(9):4214-4234

47. Chavoshi SZ, Xu S, Luo X (2016) Dislocation-mediated plasticity in silicon during nanometric cutting: a molecular dynamics simulation study. Mater Sci Semicond Process 51:60-70

48. Chavoshi SZ, Luo X (2016) Molecular dynamics simulation study of deformation mechanisms in 3C-SiC during nanometric cutting at elevated temperatures. Mater Sci Eng A 654:400-417

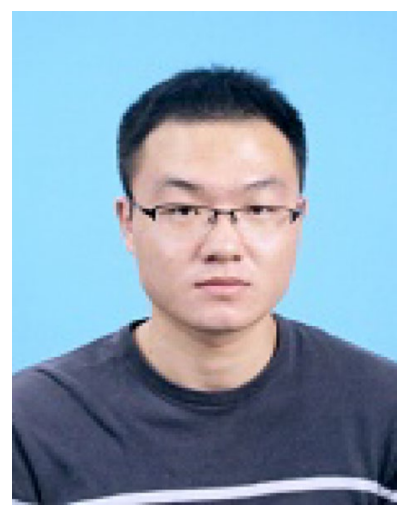

Pengfei Fan is a Doctoral candidate of DMEM at the University of Strathclyde, UK. His research interests include ultra precision manufacturing, nanofabrication and multiscale simulation technique.

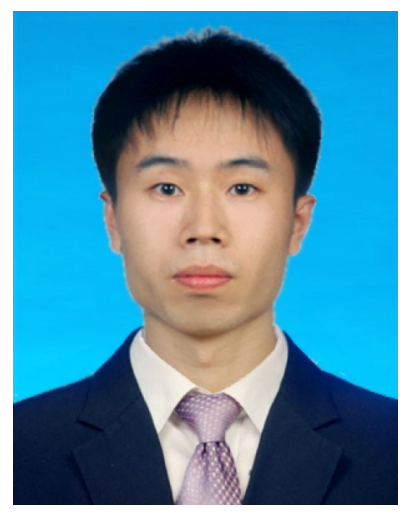

Fei Ding is a Doctoral candidate of DMEM at the University of Strathclyde, UK. His research interests include precision motion control, advanced manufacturing techniques and ultraprecision machine tool design.

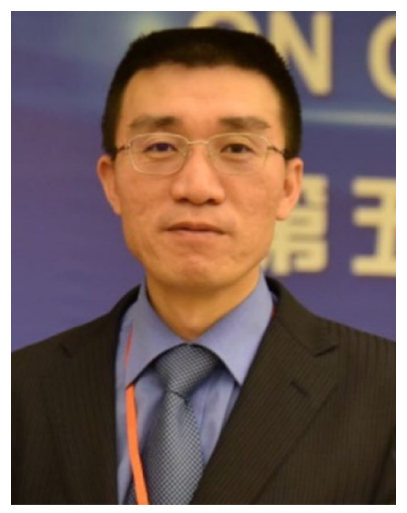

Dr. Xichun Luo is a Professor in ultra precision manufacturing and technical director of Centre for Precision Manufacturing at the University of Strathclyde. He is Fellow of the International Society for Nanomanufacturing. His research interests include ultra precision machining, micromachining and nanomanufacturing with more than 100 papers in peer-reviewed journals and conferences.

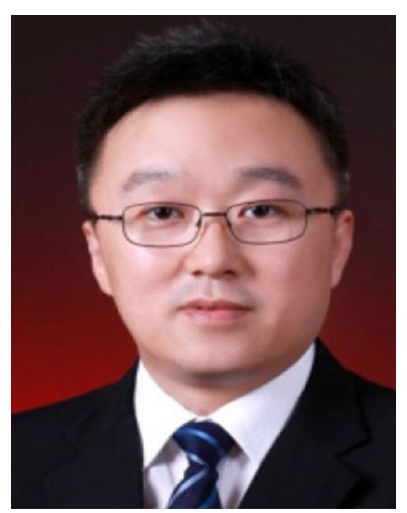

Dr. Yongda Yan is a professor in School of Mechatronics Engineering, Center for Precision Engineering and Key Laboratory of Micro-systems and Microstructures Manufacturing of Ministry of Education of Harbin Institute of Technology. He is a research affiliate of CIRP since 2013. His main research field is the micro/nano mechanical machining techniques and their applications. He received his M.S. and Ph.D. degrees in mechanical engineering from Harbin Institute of Technology 


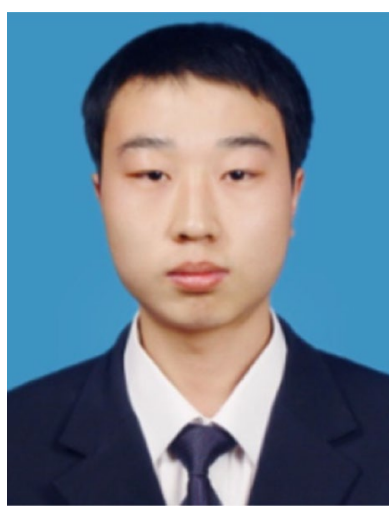

Dr. Yanquan Geng is an associate professor in Center for Precision Engineering and Key Laboratory of Micro-systems and Microstructures Manufacturing of Ministry of Education of Harbin Institute of Technology. He received his B.S., M.S. and Ph.D. degrees in mechanical engineering from Harbin Institute of Technology in 2009, 2011 and 2016. After joining Prof. Yongda Yan's group since 2011, he started his research on the AFM-based nanomachining technique and related

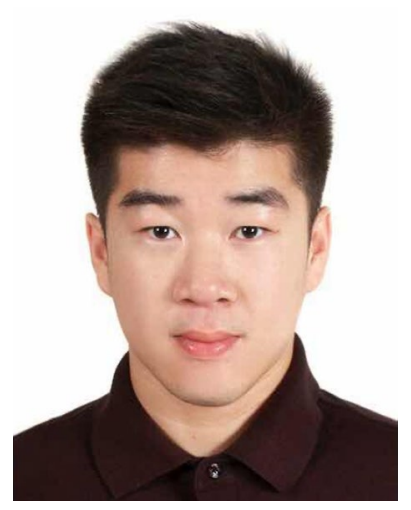

Yuzhang Wang is a Doctoral candidate of school of mechanical engineering at the Harbin Institute of Technology. His research interests include nanofabrication, precision manufacturing techniques and system control.

applications 